\title{
Solid rectal ulcer syndrome: An unusual case presentation
}

Nikolaos Chrysanthos ${ }^{1 *}$, Paraskevi Alexandrou ${ }^{2}$ and Andreas Lazaris ${ }^{2}$

${ }^{1}$ Gen Hospital of Agios Nikolaos, Crete, Greece

${ }^{2}$ Laiko Hospital, Athens, Greece

Polyps of the gross intestine are easily detected during colonoscopies and usually been excluded at the same time. Histology usually describes adenomas with dysplasia and rarely hyperplasia or inflammation.

A solitary rectal ulcer is a benign lesion characterized by rectal bleeding, tenesmus, feeling of incomplete evacuation, and rarely by abdominal pain, proctalgia, or defecation urgency. Several causes have been associated with the presence of SRUS: constipation, rectal trauma, radiotherapy, ergotamine suppositories, etc.

We present an unusual case of a 59 years old female patient with irritable bowel syndrome, and arthritis who underwent a screening colonoscopy. In the rectosigmoid junction a hemorrhagic polypoid mass with a diameter of $1.5 \mathrm{~cm}$ (Figure 1), has been detected and been excluded. Histology reveals a solid rectal ulcer characterized by abnormal hyperplastic crypts, dense mixed type inflammatory infiltrates of the lamina propria and ulceration on its surface.

SRUS usually presents ulceration of the mucosa with rectal bleeding or tenesmus. However, our patient had a unique presentation and a lack of symptoms. We repeat endoscopy three months later and mucosa in the rectosigmoid junction was normal (Figure 2). No other medication has been used.

Although these lesions are typically located in the rectum and bleeds, they rarely might be presented as large polyps.

Rejection is the treatment of choice.

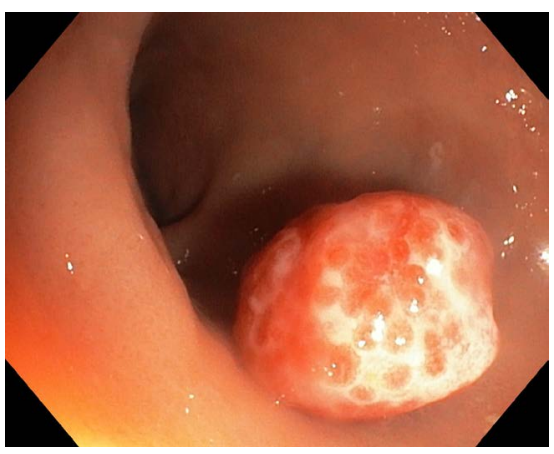

Figure 1

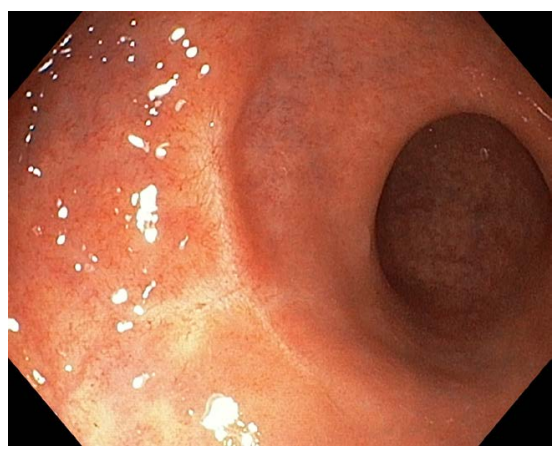

Figure 2
Copyright: $(12020$ Chrysanthos N. This is an open-access article distributed under the terms of the Creative Commons Attribution License, which permits unrestricted use, distribution, and reproduction in any medium, provided the original author and source are credited.
${ }^{\star}$ Correspondence to: Nikolaos Chrysanthos, Gen Hospital of Agios Nikolaos, Crete, Greece, Tel: +302841343000; E-mail: nchrysanthos@yahoo.gr

Received: February 05, 2020; Accepted: February 21, 2020; Published: February 24,2020 